\title{
Broad bandwidth waveguide polarizer via grating mediated mode conversion
}

\author{
Jun Zheng, ${ }^{1}$ Zhi-Cheng Ye, ${ }^{2,}$ Cheng-Liang Wang, ${ }^{2}$ Yi-Fei Fu, ${ }^{2}$ Zheng- \\ MING SHENG ${ }^{1,3}$
}

${ }^{1}$ Key Laboratory for Laser Plasmas (Ministry of Education) and School of physics and astronomy, Collaborative Innovation Center of IFSA (CICIFSA), Shanghai Jiao Tong University, Shanghai, 200240, China

${ }^{2}$ National Engineering Laboratory for TFT-LCD Technology, Department of Electronic Engineer, Shanghai Jiao Tong University, Shanghai, 200240, China

${ }^{3} S U P A$ and Department of Physics, University of Strathclyde, Glasgow G4 ONG, UK

*yzhch@sjtu.edu.cn

\begin{abstract}
A polarization beam splitter (PBS) based on a four-layer slab waveguide is proposed, where a sub-wavelength grating is embedded between the waveguide core and cladding. This grating not only affords Bragg momentum to tune the propagation constant of guiding modes, but also converts the forward zero order waveguide mode to the backward first one for a specific polarization. Thus, the incident light with polarization that satisfies the phase-matching condition is highly reflected in the waveguide, while other light with orthogonal polarization keeps intact and passes through it efficiently. Numerical simulations show that one can make the compact PBS for both polarizations with extinction ratio higher than $35 \mathrm{~dB}$, waveband larger than $80 \mathrm{~nm}$, grating period tolerance of $20 \mathrm{~nm}$, and waveguide height tolerance of $80 \mathrm{~nm}$. The revealed mode conversion mechanism via the sub-wavelength grating enriches the design of PBSs for integrated silicon waveguide chips.
\end{abstract}

() 2018 Optical Society of America

\section{Introduction}

Polarization beam splitters (PBSs) in the silicon-on-insulator platform are essential polarization-handling devices in increasingly attractive areas such as integrated photonic quantum circuits [1,2] and coherent optical communications [3-5]. These devices are desired to have small dimension and large fabrication tolerance. Up to now, PBSs based on different configurations have been proposed, such as directional couplers (DCs) [6-9], Mach-Zehnder interference (MZI) couplers [10], multimode interference (MMI) couplers [11, 12], photoniccrystals (PCs) $[13,14]$ and grating structures $[15,16]$. Among them, DCs and MZIs are more popular due to their simple structures and easiness of design. However, they rely on the fine resonant evanescent coupling between two very closed waveguides, thus PBSs based on them have narrow bandwidths and limited fabrication tolerance. Self-imaging interference based MMI PBSs offer excellent tolerance to wavelength variations and fabrication errors, but their size is relatively large at the millimeter scale due to the intrinsic weak polarization dependence. PCs typed PBSs, which work based on polarization dependent photonic band gaps, have high extinction ratio and broadband, while their complex structures are difficult to fabricate. Furthermore, PCs typed devices usually introduce a relatively large loss due to the scattering.

In this paper, we propose a novel PBS based on grating mediated mode conversion. It is the grating, as a layer in a four-layer slab waveguide (FLSW) $[17,18]$, that induces the mode conversion via the mechanism of grating diffraction to reflect back the TM- or TE-polarized light. Numerical simulation shows this compact PBS facilitates broadband, high polarization extinction ratio (PER) and large fabrication tolerance.

\section{Device Design and Characteristics}


A schematic of the proposed PBS device is presented in Fig. 1. The Si grating and substrate waveguide are surrounded by $\mathrm{SiO}_{2}$ cladding, which is regarded as a FLSW with region labeled with I, II, III and IV, assuming a large width along the $\mathrm{z}$ axis. The polarized light is incident into the Si waveguide core with the height of $h_{1}$. Under the mode conversion by the grating with period of T and height of $h_{2}$, TM- or TE-polarized light is reflected in other mode or transmitted through the device, depending on the device structure parameters.

Based on the wave equations in the regions and boundary conditions at the interfaces between the adjacent regions, the eigen mode equation [19] of FLSWs can be derived:

$$
k_{1} h_{1}=m \pi+\arctan \left(c_{14} \frac{p_{4}}{k_{1}}\right)+\arctan \left(c_{12} \frac{p_{2}}{k_{1}} \delta\right)
$$

where

$$
\delta= \begin{cases}\frac{p_{3}+c_{32} p_{2} \tanh \left(p_{2} h_{2}\right)}{c_{32} p_{2}+p_{3} \tanh \left(p_{2} h_{2}\right)}, \quad n_{2} \leq N \leq n_{1} \\ \frac{p_{3}-c_{32} k_{2} \tan \left(k_{2} h_{2}\right)}{c_{32} k_{2}+p_{3} \tan \left(k_{2} h_{2}\right)}, \quad n_{3} \leq N \leq n_{2}\end{cases}
$$

$m$ is the mode order; $k_{i}=k_{0} \sqrt{n_{i}^{2}-N^{2}}$,where $i=1$ or $2, p_{i}=k_{0} \sqrt{N^{2}-n_{i}^{2}}$, where $i=2,3$ or 4 , $k_{0}=2 \pi / \lambda$ is the wave number of the launched light in free space, $N$ is the mode effective index. The refractive indices of the four layers: $n_{1}=n_{S i}=3.477, n_{3}=n_{4}=n_{\mathrm{SiO}_{2}}=1.444$, $n_{2}=\sqrt{f_{\mathrm{Si}_{i}} n_{\mathrm{Si}}^{2}+f_{\mathrm{SiO}_{2}} n_{\mathrm{SiO}_{2}}^{2}}$ for TE-polarized light and $1 / \sqrt{f_{\mathrm{Si}} / n_{\mathrm{Si}}^{2}+f_{\mathrm{SiO}_{2}} / n_{\mathrm{SiO}_{2}}^{2}}$ for TM-polarized light when the grating period is much smaller than the light wavelength [20], where duty ratio $f_{S i}=t / T=0.5$ and $f_{S_{i O}}=1-f_{S i}, t$ is the width of the Si strip in each grating period. Also in Eq. (1), $c_{i j}=n_{i}^{2} / n_{j}^{2}$ for TM-polarized light and 1 for TE-polarized light. The wave vector along the propagation direction $\beta= \pm N / k_{0}= \pm \sqrt{k_{0}^{2}-k_{i}^{2}}$, where the signs of " + " and “_” correspond to the propagation directions along $+\mathrm{x}$ and $-\mathrm{x}$ axis, respectively.

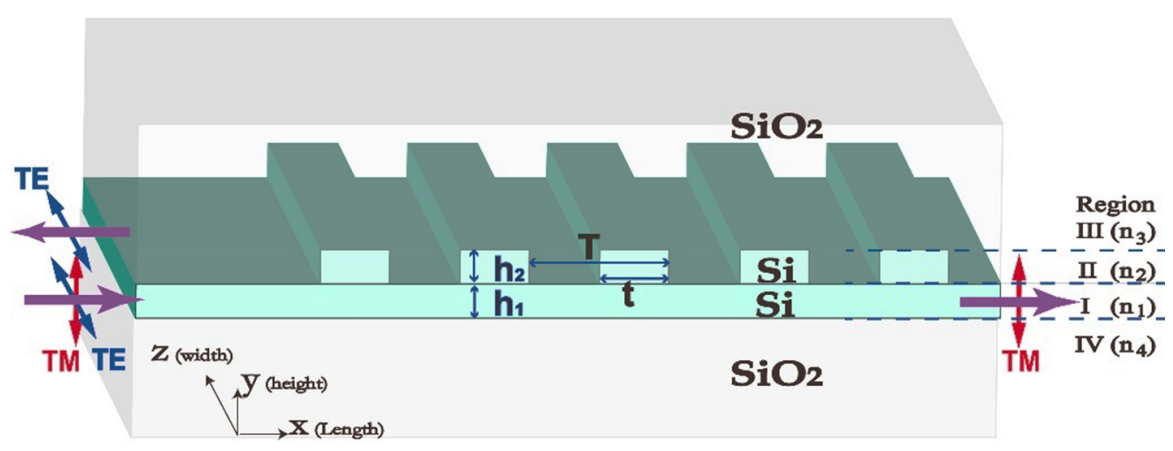

Fig. 1. Schematic of the proposed device acting as a PBS.

Figure 2(a) shows the dependence of $\beta$ on the grating height $h_{2}$. The device structure parameters are $\lambda=1.55 \mu \mathrm{m}, h_{1}=0.3 \mu \mathrm{m}$ and $f_{S i}=0.5$. The blue lines in Fig. 2(a) correspond to the $0^{\text {th }}$ modes for TE- (solid lines) and TM-polarized (dashed lines) light, where the electric-magnetic field sinusoidally oscillates in $\mathrm{Si}$ waveguide core (Region I) and 
exponentially decays in other regions. The red lines correspond to the $1^{\text {st }}$ modes, where electric-magnetic field sinusoidal oscillations in the Si waveguide core and $\mathrm{Si}$ grating layer (Region I and II) while exponentially decays in other regions.

The Si grating can induce mode conversion from $\beta_{m_{1}}$ to $\beta_{m_{2}}$ by the Bloch's wave vector $k_{g}=2 \pi / T$, following the equation $\beta_{m_{2}}=\beta_{m_{1}}-k_{g}$. For grating with pitch $T=0.3 \mu \mathrm{m}$ and $k_{g}=5.17 k_{0}$ the line with $\beta_{0}-k_{g}$ for TE- and TM-polarized light is shown with solid and dashed thin blue lines in Fig. 2(a), respectively. There is a cross point $\beta_{-1}=-2.06 k_{0}$ at $h_{2}=0.155 \mu \mathrm{m}$ for TE-polarized light and no cross points for TM-polarized light. Thus, the grating with $T=0.3 \mu \mathrm{m}$ can change the TE light from the mode $\beta_{0}=3.11 k_{0}$ to the mode $\beta_{-1}=-2.06 k_{0}$, which is shown by a vertical black arrow in Fig. 2(a). The corresponding electric field distribution $E_{x}(y)$ of the launched light with $0^{\text {th }}$ mode and the reflected light with $1^{\text {st }}$ mode are presented in Fig. 2(b) with solid blue and red lines, respectively. Meanwhile, TM-polarized light with $0^{\text {th }}$ mode cannot be converted to other modes by this grating. Thus, only TE-polarized light can be reflected backward, while TM-polarized light can pass through this FLSW. The transmitted spectra and snapshots of field distributions obtained by twodimensional finite difference time domain (2D FDTD) simulation for TE- and TM-polarized light are presented in Fig. 2(c) and 2(d), respectively The TE transmission is around $-40 \mathrm{~dB}$ for the wavelength from $1.54 \mu \mathrm{m}$ to $1.62 \mu \mathrm{m}$, while TM transmission is $0 \mathrm{~dB}$. The TM field distributions show that the TM-polarized light transfers directly to the output-port, rarely affected by the grating. While the TE-polarized light is reflected back to the input-port with mode field changed from bottom to upper and down positions corresponding to the $1^{\text {st }}$ order mode. The simulation results agree with the theory analysis well.

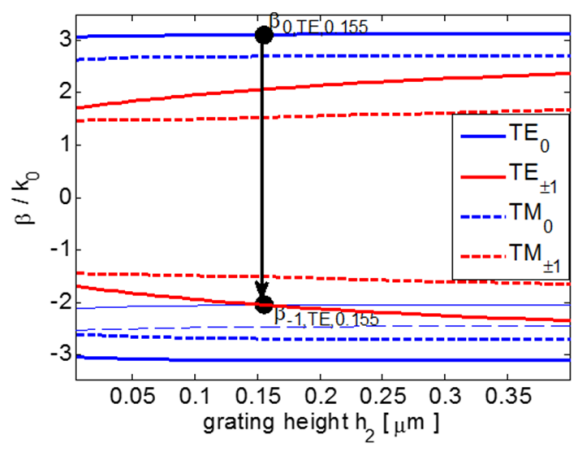

(a)

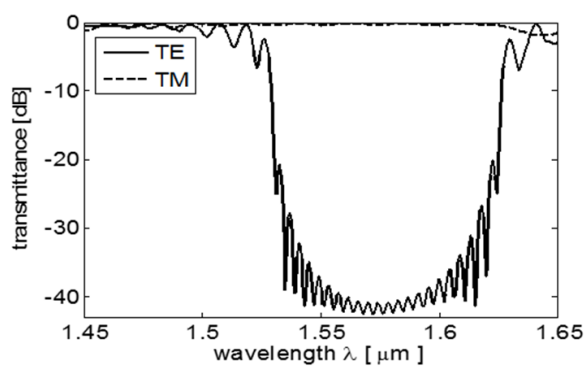

(c)

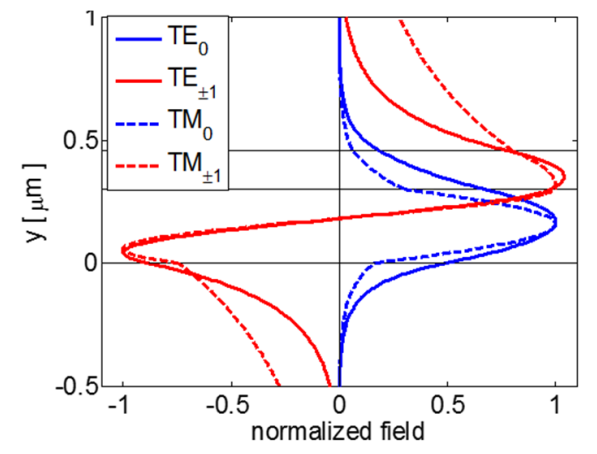

(b)

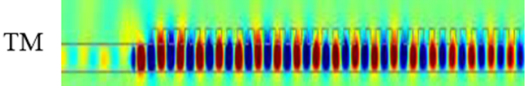

TE

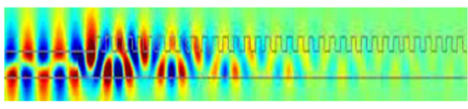

(d)

Fig. 2. (a) The wave vector along the propagation direction $\beta$ supported in FLSW for TE (solid lines) and TM (dashed lines) mode. The red and blue lines correspond to the $0^{\text {th }}$ and $1^{\text {st }}$ mode, 
respectively. (b) Field distribution $E_{z}(y)$ (solid lines) and $H_{z}(y)$ (dashed lines) for the $0^{\text {th }}$ (blue lines) and $1^{\text {st }}$ (red lines) mode. (c) TE (solid line) and TM (dashed line) transmitted spectra. (d) Snapshots of field distributions of $E_{z}$ for TE-polarized light and $H_{z}$ for TM-polarized light. The common structure parameters: waveguide core height $h_{1}=0.3 \mu \mathrm{m}$, grating height $h_{2}=0.155 \mu \mathrm{m}$, grating pitch $T=0.3 \mu \mathrm{m}$, grating total length in $x$ axis is $16 \mu \mathrm{m}$, wavelength $\lambda=1.55 \mu \mathrm{m}$.

Figure 3(a) shows the grating pitch $T$ inducing the mode conversion for different grating height $h_{2}$ and light polarization state. The transmitted TE-to-TM PER changing with $T$ for $h_{2}=0.155 \mu \mathrm{m}$ (the dotted line in Fig. 3(a)) is presented in Fig. 3(b), where, the negative PER corresponds to the TE-reflected and TM-transmitted PBS and the positive PER corresponds to the TM-reflected and TE-transmitted PBS. It shows that the grating with different pitch $T$ can produce different type of PBS. For the optical communication C-band wavelength ranges from $1.53 \mu \mathrm{m}$ to $1.56 \mu \mathrm{m}, T$ should be $0.282 \sim 0.296 \mu \mathrm{m}$ for reflecting TE-polarized light and transmitting TM-polarized light with the absolute value of PER larger than $30 \mathrm{~dB}$ as shown with the dark blue strip in Fig. 3(b), where TE mode is converted from $0^{\text {th }}$ to $1^{\text {st }} ; T$ should be $0.338 \sim 0.346 \mu \mathrm{m}$ for reflecting TM-polarized light and transmitting TE-polarized light with PER larger than $18 \mathrm{~dB}$ as shown with the dark red strip in Fig. 3(b), where TM mode is converted from $0^{\text {th }}$ to $1^{\text {st }}$. These optimized periods are around the analysis results shown in Fig. 3(a). The slight difference mainly comes from the estimate of refractive index of the grating layer. In theory, the TE $0^{\text {th }}$ mode can be coupled to the $0^{\text {th }}$ mode with the opposite propagation direction by the grating with $T=0.25 \mu \mathrm{m}$, as shown with the solid blue line in Fig. 3(a). However, the field distribution for the $0^{\text {th }}$ mode is mainly in the $\mathrm{Si}$ waveguide core (Region I) instead of the grating layer (Region II), as shown with the blue lines in Fig. 2(b), thus the grating's influence to the mode conversion from $0^{\text {th }}$ to $0^{\text {th }}$ is relatively weak and the corresponding absolute value of PER is low, especially for TM-polarized light. We almost cannot see the case of TM mode conversion from $0^{\text {th }}$ to $0^{\text {th }}$ in Fig. 3(b). For the TE case, the absolute value of PER is only around $13 \mathrm{~dB}$ in the entire $\mathrm{C}-$ band wavelength range as shown with the light blue strip in Fig. 3(b).

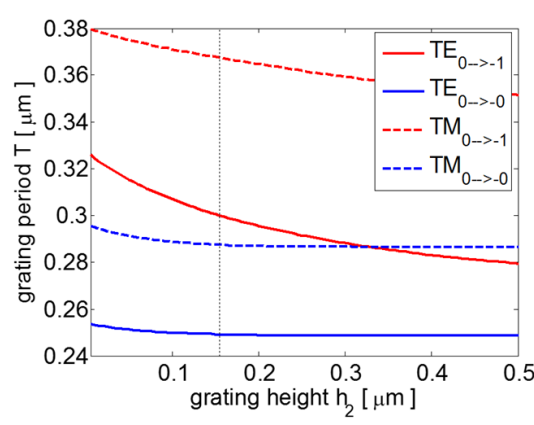

(a)

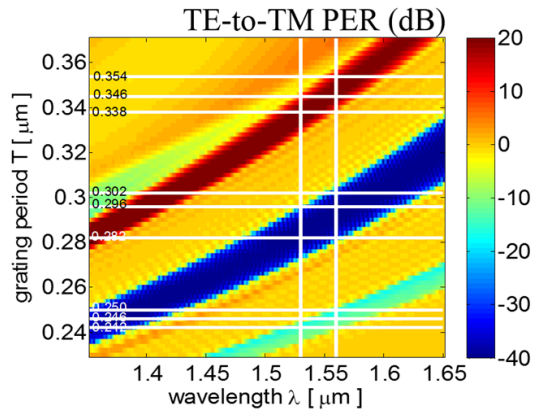

(b)

Fig. 3. (a) Grating pitch $\mathrm{T}$ to converse the $0^{\text {th }}$ mode to reflected $0^{\text {th }}$ or $1^{\text {st }}$ mode for TE (solid lines) and TM (dashed lines) light, respectively. (b) The transmitted TE-to-TM PER for different wavelength and grating pitch $\mathrm{T}$. The two white vertical lines show the C-band wavelength range. The common structure parameters are same as that in Figure 2.

The device length is a key parameter for PBS, which stands for the compactness of device. The transmission changing with the grating length along the $x$ axis is shown in Fig. 4(a). The longer the grating is, the larger absolute value of PER is. For the wavelength of 1.55 $\mu \mathrm{m}$, the PER is $-32 \mathrm{~dB}$ in the case of grating longer than $10 \mu \mathrm{m}$ and it exceeds $-40 \mathrm{~dB}$ when grating is longer than $13 \mu \mathrm{m}$. 
The transmission changing with the device width in the $z$ axis by three-dimensional simulation is shown in Fig. 4(b). With the increase of the width, the spectra tend to be stable gradually and converge. For the same grating length, the transmittance for the device width of $21 \mu \mathrm{m}$ (red line in Fig. 4(b)) is almost same with the results by 2D simulation (red line in Fig. 4(a)). Hence, it is reasonable to investigate the characteristics of the device by $2 \mathrm{D}$ simulation for the device width larger than $21 \mu \mathrm{m}$. It can considerably save computer resource, while maintaining the validity of the simulation results.

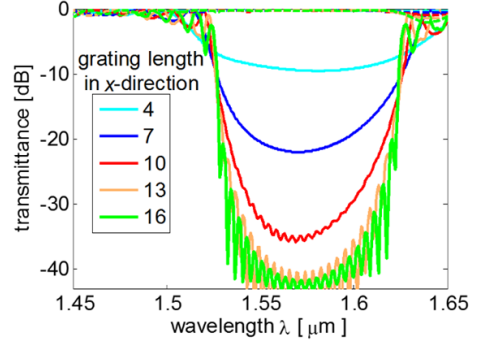

(a)

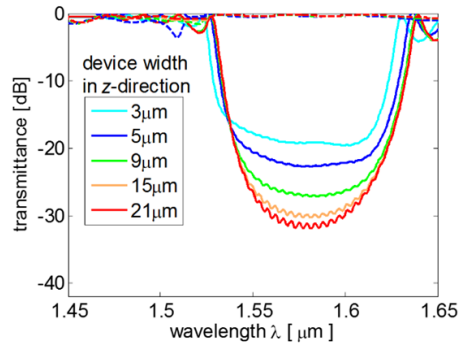

(b)

Fig. 4. TM (solid line) and TE (dashed line) transmitted spectra for (a) different grating length by 2D FDTD simulation and (b) different device width in $\mathrm{z}$ axis by 3D FDTD simulation. The common structure parameters: waveguide core height $h_{1}=0.3 \mu \mathrm{m}$, grating height $h_{2}=0.155 \mu \mathrm{m}$ and grating period $T=0.3 \mu \mathrm{m}$. The grating length is $10 \mu \mathrm{m}$ in (b) for saving the computer resource.

\section{Effect of Waveguide Parameters}

The effects of the waveguide parameters including the core height $h_{1}$, grating height $h_{2}$, grating pitch $T$, duty ratio $f_{S i}$ and refractive index $n_{\text {grating }}$ are investigated. Figure 5(a) shows that $h_{1}$ and $h_{2}$ have tolerance lower than $0.08 \mu \mathrm{m}$. For example, for $h_{1}=0.20 \mu \mathrm{m}, h_{2}$ could be chose in the range of $0.22 \sim 0.30 \mu \mathrm{m}$ for TE-reflected PBS in entire optical communication $\mathrm{C}-$ band wavelength range with TM-to-TE PER larger than $30 \mathrm{~dB}$. When $h_{1}=0.35 \mu \mathrm{m}, h_{2}$ could be chose in the range of $0.26 \sim 0.37 \mu \mathrm{m}$ for TM-reflected PBS in C-band with TE-to-TM PER larger than $30 \mathrm{~dB}$. These results show that the optimized $h_{1}$ and $h_{2}$ in TM-reflected PBS are both larger than the TE-reflected PBS case, which is due to the less effective index in TM mode, as shown in Fig. 2(a). Figures 5 and 3(b) also shows that one can find a combination of $h_{1}, h_{2}, T$ and $f_{S i}$ to satisfy the momentum-matching condition to realize TE-reflected or TMreflected PBS for the expected wavelength with good fabrication tolerance. Generally, to get PER larger than $30 \mathrm{~dB}$ for $\lambda=1.55 \mu \mathrm{m}$, TE-reflected PBS can be produced with structure parameters in the range of $h_{1}=0.2 \sim 0.3 \mu \mathrm{m}, h_{2}=0.10 \sim 0.32 \mu \mathrm{m}$; TM-reflected PBS can be produced with $h_{1}=0.3 \sim 0.35 \mu \mathrm{m}, h_{2}=0.28 \sim 0.40 \mu \mathrm{m}$, under the condition of grating longer than $16 \mu \mathrm{m}$ and device width larger than $21 \mu \mathrm{m}$, where $T=0.3 \mu \mathrm{m}$ with tolerance of $0.02 \mu \mathrm{m}$ as shown in Fig. 3(b) and $f_{S i}=0.3 \sim 0.5$ as shown in Fig. 5(b).

The grating could be other materials with different $n_{\text {grating, }}$, whose effect on the operating spectra of the PBS is shown in Fig. 5(c). With the increase of $n_{\text {grating }}$ from 2.5 to 4, the bandwidth of TE-reflected PBS with TE-to-TM PER larger than $10 \mathrm{~dB}$ increases from 20 to $140 \mathrm{~nm}$. However the central wavelength also changes from 1.51 to $1.63 \mu \mathrm{m}$. The effect of $n_{\text {grating }}$ on the bandwidth of TE-reflected PBS is stronger than TM-reflected PBS case. 


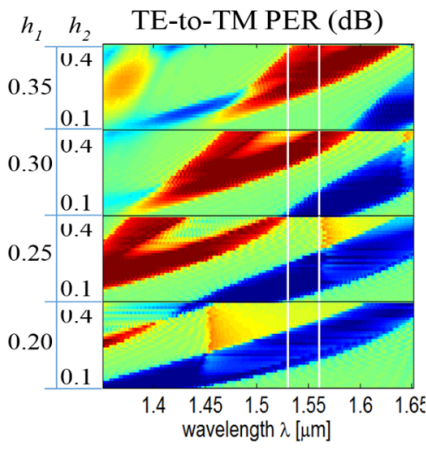

(a)

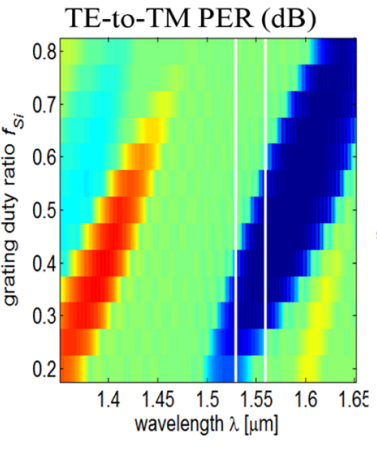

(b)

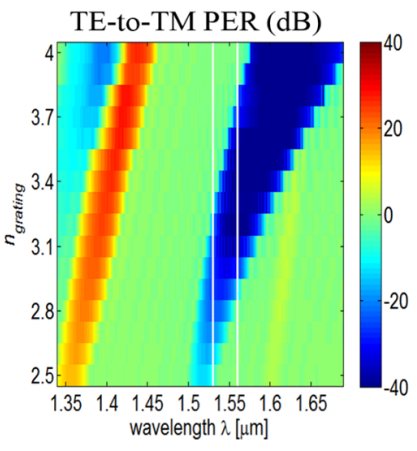

(c)

Fig. 5. Transmitted TE-to-TM PER for different waveguide height $h_{1}$ and grating height $h_{2}$ (a), grating duty ratio $f_{S i}$ (b) and grating refractive index $n_{\text {grating }}(\mathrm{c})$. The two white vertical lines show the C-band. The common structure parameters are same as that in Figure 2.

\section{Conclusions}

A waveguide PBS based on mode conversion in a FLSW is proposed. The grating inserted between the waveguide core and cladding affords the Bragg momentum to tune the propagation constant of the guiding mode and make one polarized light reflected with changed order of the waveguide mode. While the other orthogonal polarized light is transmitted efficiently. One can engineer the layered structure with appropriate $\mathrm{Si}$ waveguide core height $h_{1}$, Si grating height $h_{2}$ and period $T$ to satisfy the momentum-matching condition to realize TE-reflected or TM-reflected PBS for the expected wavelength. Simulation indicates that the grating longer than $16 \mu \mathrm{m}$ and the device wider than $21 \mu \mathrm{m}$ are needed to make PER over $40 \mathrm{~dB}$. The tolerances of T, $h_{1}$ and $h_{2}$ are relaxed to 20,80 and $80 \mathrm{~nm}$, respectively. The bandwidth is around $80 \mathrm{~nm}$. The proposed structure has a broad prospect in polarization maintenance required by optical interconnection. The revealed mechanism of grating mediated mode conversion enriches the design of waveguide integrated PBS for silicon optical chips.

Funding. Natural National Science Foundation of China (NSFC) $(61775136,11721091)$.

\section{References}

1. C. Schaeff, R. Polster, R. Lapkiewicz, R. Fickler, S. Ramelow, and A. Zeilinger, "Scalable fiber integrated source for higher dimensional path-entangled photonic quNits," Opt. Express 20(15), 16145-16153 (2012).

2. L. Sansoni, F. Sciarrino, G. Vallone, P. Mataloni, A. Crespi, R. Ramponi, and R. Osellame, "Polarization entangled state measurement on a chip," Phys. Rev. Lett. 105(20), 200503 (2010).

3. G.F. Li, "Recent advances in coherent optical communication," Advances in Optics and Photonics 1, 279-307 (2009).

4. Y. Han and G.F. Li, Coherent optical communication using polarization multiple-input-multiple-output. Opt. Express 13(19), $7527-5734$ (2005).

5. D. Dai, L. Liu, S. Gao, D. X. Xu and S. He, "Polarization management for silicon photonic integrated circuits," Laser Photonics Rev. 7(3), 303-328 (2013).

6. Z. Zhang, X. Hu and J. Wang, “On-chip optical mode exchange using tapered directional coupler," Sci. Rep. 5, 16072 (2015).

7. Y.L. Xiong, J.G. Wangüemert-Pérez, D.X Xu, J. H. Schmid, P. Cheben and W.N. Ye, "Polarization splitter and rotator with subwavelength grating for enhanced fabrication tolerance," Opt. Lett. 39(24), 6931-6934 (2014).

8. K. Tan, Y. Huang, G.Q. Lo, C.K. Lee and C.Y. Yu, "Compact highly-efficient polarization splitter and rotator based on $90^{\circ}$ bends," Opt. Exp. 24(13) 14506 (2016). 
9. C. Liu, L.S. Yan, A.L. Yi, H.Y. Jiang, Y. Pan, L. Jiang, X. Feng, W. Pan and B. Luo, "Ultrahigh suppression broadband polarization splitter based on an asymmetrical directional coupler," IEEE Photon. Jour. 9(5), 6601709 (2017)

10. D. Dai, Z. Wang, and J.E. Bowers, "Considerations for the design of asymmetrical Mach-Zehnder interferometers used as polarization beam splitters on a submicrometer silicon-on-insulator platform,” J. Ligh. Tech., 29(12), 1808-1817 (2011).

11. L.B. Soldano and E.C.M. Pennings, "Optical multi-mode interference devices based on self-imaging: principles and applications," Journal of Ligh. Tech. 13(4), 615-627 (1995).

12. O. Bryngdahl, "Image formation using self-imaging techniques," J. Opt. Soc. A. 63(4), 416-419 (1973).

13. Y. Zhang, H.X. Lei and B.J. Li, "Polarization beam splitter with wide bandwidth in air-hole-based periodic dielectric waveguides," Opt. Comm. 283, 2140-2145 (2010).

14. F. Lu, F. Xiao, K. Li, Z.H. Wang and A.S. Xu, "Bidirectional Polarization Splitting Couplers Based on Dielectric-Coated Metallic Gratings," IEEE Phot. Tech. Lett. 25(17), 1695-1698 (2013).

15. J.B. Feng, Z.P. Zhou, "Polarization beam splitter using a binary blazed grating coupler," Opt. Lett. 32(12), 1662-1664 (2007)

16. R. Halir, P.J. Bock, P. Cheben, A. Ortega-Monux, C. Alonso-Ramos, J.H. Schmid, J. Lapointe, D.X. Xu, J. G. Wanguemert-Perez, I. Molina-Fernandez and S. Janz, "Waveguide sub-wavelength structures: a review of principles and applications," Laser Photon. Rev. 9(1), 25-49 (2015).

17. M. Kudo and Y. Mushiake, "Mode selecting characteristics of four-layer dielectric slab waveguide," Radio Sci. 17(1), 125-134 (1982).

18. I. S. Panyaev, N. N. Dadoenkova, Y. S. Dadoenkova, I. A. Rozhleys, M. Krawczyk, I. L. Lyubchanskii and D. G. Sannikov, "Optical properties of a four-layer waveguiding nanocomposite structure in near-IR regime," Opt. Quant. Electron. 48(12), 556 (2016).

19. K. Okamoto, Fundamentals of optical waveguides (Academic, 2000).

20. S.M. Rytov, "The electromagnetic properties of finely layered medium," Sov. Phys. JETP 2, $466-475$ (1956).

\section{Article thumbnail:}

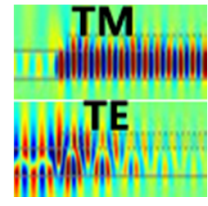

Abstract Thumbnail (100 pix * 100 pix)

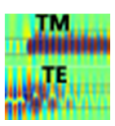

ToC Thumbnail (50 pix* 50 pix) 